EPJ Web of Conferences 41, 03001 (2013)

DOI: $10.1051 /$ epjconf/20134103001

(C) Owned by the authors, published by EDP Sciences, 2013

\title{
The Earliest Stage of Photoinduced Phase Transition in a Strongly Correlated Organic System Using a 10-fs Pulse
}

\author{
Ken Onda ${ }^{1,2}$, Yoshitaka Matsubara ${ }^{3}$, Tadahiko Ishikawa ${ }^{3}$, Yoichi Okimoto ${ }^{3}$, Shin-ya Koshihara S, $^{3,4}$ \\ Takaaki Hiramatsu ${ }^{5}$, Gunzi Saito ${ }^{5}$, Yoshiaki Nakano ${ }^{6}$, Hideki Yamochi ${ }^{6}$ \\ ${ }^{1}$ Interactive Research Center of Science, Tokyo Institute of Technology, Nagatsuta, Midori, \\ Yokohama 226-8502, Japan \\ ${ }^{2}$ PRESTO, Japan Science and Technology Agency (JST) \\ ${ }^{3}$ Department of Chemistry and Materials Science, Tokyo Institute of Technology, O-okayama, \\ Meguro, Tokyo 152-8551, Japan \\ ${ }^{4}$ CREST, Japan Science and Technology Agency (JST) \\ ${ }^{5}$ Faculty of Agriculture, Meijo University, Tempaku-ku, Nagoya 468-8502, Japan \\ ${ }^{6}$ Research Center for Low Temperature and Materials Sciences, Kyoto University, Sakyo-ku, Kyoto \\ 606-8501, Japan
}

\begin{abstract}
We examined the initial excited state before photoinduced phase transition in a charge transfer complex (EDO-TTF $)_{2} \mathrm{PF}_{6}$ and revealed the conversion process to the photoinduced phase and the electronic coherence at the excited state.
\end{abstract}

\section{Introduction}

Strongly correlated systems exhibit a wide variety of phases such as Mott insulator, charge density wave insulator, ferromagnetic metal, superconductor due to strong electron-electron interaction [1]. Organic charge transfer (CT) complexes consisting of $\pi$-electron molecules are essentially strongly correlated systems because the Coulomb interactions between onsite/offsite electrons are comparable to the transfer energy between constituent molecules [2, 3]. In addition, the structures of CT complexes are soft and flexible because the molecules are bound to each other by relatively weak cohesive forces; thus, CT complexes easily undergo phase transition by weak external stimuli. Photoirradiation is one of the ways to induce the phase transition in this type of materials and the phenomenon is called photoinduced phase transition (PIPT). By PIPT, macroscopic physical properties can be changed quickly and cooperatively, so that the mechanism has been attracting the attention of many researchers. However, the PIPT process is so fast that it is difficult to see the states before emergence of photoinduced phase. In this paper, we studied the earliest stage of PIPT in a quasi-one-dimensional (1D) CT complex, (EDO-TTF $)_{2} \mathrm{PF}_{2}$, using an intensive 10 -fs pulse, and revealed how the photoinduced phase is created from the initial excited state.

\section{Experimental}

A 10-fs pulse was generated using a gas-filled hollow glass fiber and three types of chirped mirrors from a 120-fs output pulse of a Ti:sapphire chirped pulse amplifier [4]. The inner diameter of the

This is an Open Access article distributed under the terms of the Creative Commons Attribution License 2.0, which permits unrestricted use, distribution, and reproduction in any medium, provided the original work is properly cited. 
fiber was $200 \mu \mathrm{m}$ and the two atmospheres of Krypton gas were used for the nonlinear medium. The energy of the compressed pulse was $\sim 50 \mu \mathrm{J} /$ pulse operating at $1 \mathrm{kHz}$.

\section{Results and discussion}

(EDO-TTF $)_{2} \mathrm{PF}_{6}$ is known to show gigantic reflectivity change more than $100 \%$ immediately after photoexcitation of a CT band in the (0110) charge order insulator phase [5], where the numbers in the parenthesis represent the sequence of charge on a donor molecule in a $1 \mathrm{D}$ column. The photoinduced state at around $100 \mathrm{fs}$ was assigned to the (1010) photoinduced original phase by measuring the transient reflectivity spectrum ranging from $18 \mu \mathrm{m}$ to $590 \mathrm{~nm}$ using a 120 -fs pulse [6]. Afterward, the photoinduced phase was found to turn to the charge melting $(0.5,0.5,0.5,0.5)$ metallic phase with time constant of 94 ps by time-resolved infrared vibrational spectroscopy [7]. To observe the earliest stage of this PIPT process, we measured the reflectivity change $(\Delta R / R)$ after photoexcitation of the CT band using the 10-fs pulse. Figure 1(a) shows the static reflectivity spectra of the sample at $25 \mathrm{~K}$ and the 10 -fs pulse. The peak centered at $1.4 \mathrm{eV}$ in the sample spectrum is assigned to the CT band from (0110) to (0200). We used the whole spectrum of the 10-fs pulse for the pump and probe pulses, and the reflected probe pulse was selected using an interference filter in front of the photo-detector. Figure 1(b) shows the temporal profile of $\Delta \mathrm{R} / \mathrm{R}$ selecting the hatched region in Figure 1(a). The $\Delta \mathrm{R} / \mathrm{R}$ gradually increases after quick rise accompanied with intensity oscillation. Intriguingly, there is some signal in the negative delay region down to $-50 \mathrm{fs}$. First, we analysed the positive part of this profile using the model that the initial exited state is converted exponentially into the photoinduced phase, and obtained the time constant of $40 \mathrm{fs}$. The simulated curve using this model is shown as the purple line in Figure 1(b). The red and blue lines represent temporal variation of the initial state and the photoinduced phase, respectively. This delayed emergence of the photoinduced phase indicates that the PIPT requires a lead time to create the photoinduced phase after photoexcitation. This lead time is already predicted as the delay of charge exchange by the theoretical model calculation in our previous study [6], and the strong electronlattice interaction in this sample causes this lead time.
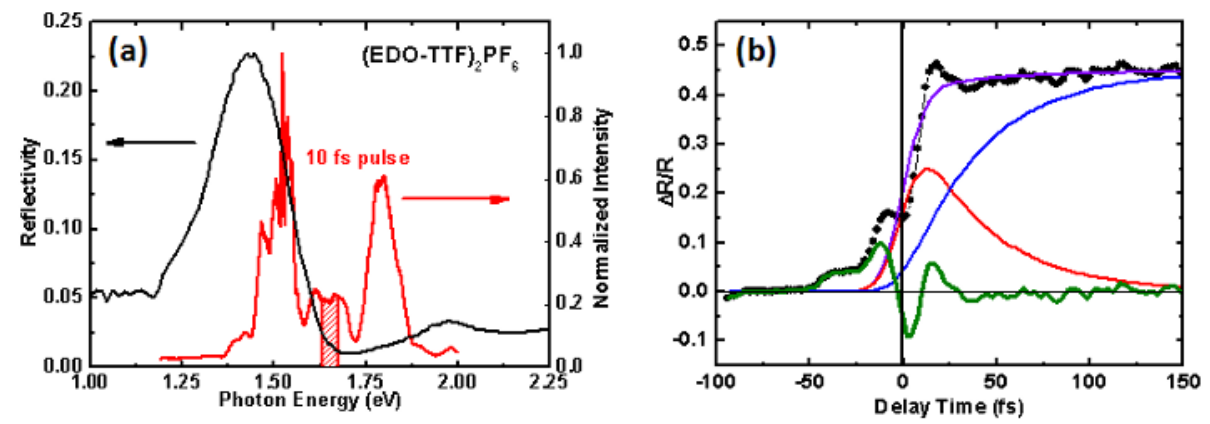

Fig. 1. (a) Spectra of (EDO-TTF) ${ }_{2} \mathrm{PF}_{6}$ (black line) and 10-fs pulse (red line). (b) Temporal profile of reflectivity change (black dots) and simulated curves (purple, blue and red lines). The green line is the redisual between the observed data and the simulated curve.

We next analysed the residual part between the observed data and the simulation shown as green line in Figure 1(b). Since the cross-correlation of the pump and probe pulses is $\sim 16 \mathrm{fs}$, the signal is obviously observed in the negative delay time region. This type of negative delay signal is sometimes seen at around zero delay in the ultrafast pump-probe measurement and is attributed to the third-order nonlinear optical process $[8,9]$. In the negative delay region, the probe pulse excites the sample first, and then the delayed pump pulse creates the transient grating during the electronic coherence induced by the probe pulse persists. By this transient grating, a part of the pump pulse is 
diffracted to the detector facing the probe pulse. Thus, the lifetime of the negative delay signal corresponds to the electronic coherence time at the excited state. Based on this model, we reproduced the negative delay signal using an exponential decay function and a trigonometric function expressing oscillation shown in Figure 2(a), and the values of $24 \mathrm{fs}$ and $38.5 \mathrm{THz}$ were obtained for the decay lifetime and the frequency of oscillation, respectively. As mentioned above, the 24-fs decay time corresponds to the electronic coherence time of the excited state, and judging from the wavenumber, the oscillation of $38.5 \mathrm{THz}=1280 \mathrm{~cm}^{-1}$ is attributed to the coherent phonon of intra-molecular vibration. In conclusion, the initial excited state is prepared on the basis of Franck-Condon principle followed by the electronic coherence of this state decays with the lifetime of $24 \mathrm{fs}$ accompanied with coherent phonon of intra-molecular vibration, and then the photoinduced phase emerges over 40 fs cooperatively. The photoinduced state relaxes over a few picoseconds accompanied with coherent phonon of inter-molecular vibration (not shown here). Figure 2(b) shows the summary of the earliest dynamics of PIPT in (EDO-TTF $)_{2} \mathrm{PF}_{6}$.

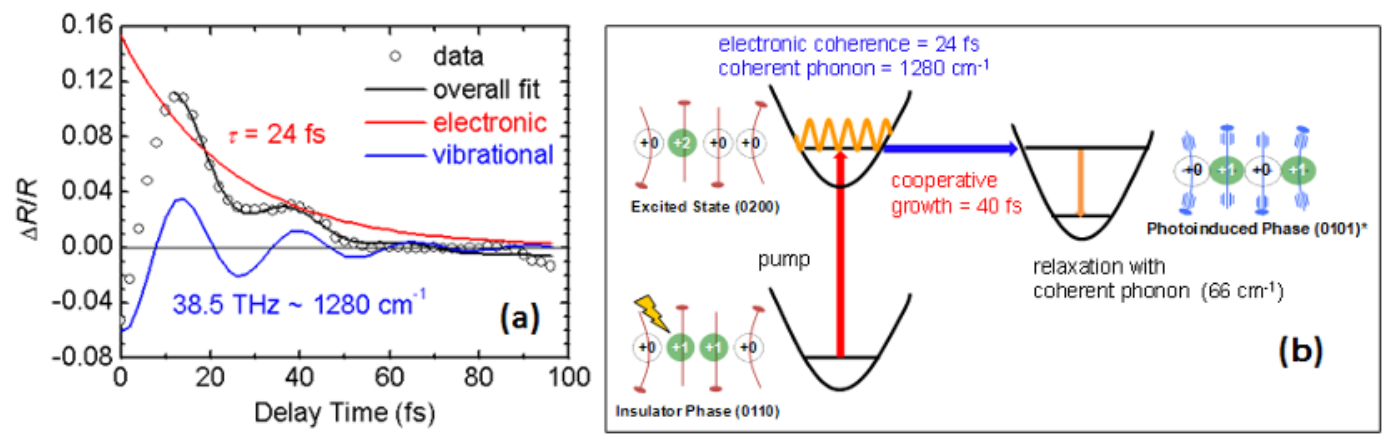

Fig. 2. (a) Analysis of the redidual part in the negative delay region (the delay time is inverse) . (b) Summary of the earliest dynamics of photoinduced phase transition in $(\mathrm{EDO}-\mathrm{TTF})_{2} \mathrm{PF}_{6}$.

\section{References}

1. E. Dagotto, "Complexity in Strongly Correlated Electronic Systems," Science, 309, 257 (2005)

2. S. Kagoshima, K. Kanoda, T. Mori, ed. "Special Topics on Organic Conductors," J. Phys. Soc. Jpn. 75 (2006).

3. S. Uji, T. Mori, T. Takahashi, ed. "Topical review on Focus on Organic Conductors," Sci. Technol. Adv. Mater. 10 (2009)

4. M. Nisoli, S. De Silvestri, and O. Svelto, "Generation of high energy 10 fs pulses by a new pulse compression technique," App. Phys. Lett. 68, 2793 (1996)

5. M. Chollet, M. Guerin, N. Uchida, et al. "Gigantic Photoresponse in 1/4-Filled-Band Organic Salt (EDO-TTF) ${ }_{2} \mathrm{PF}_{6}$," Science, 307, 86 (2005).

6. K. Onda, S. Ogihara, K. Yonemitsu, et al. "Photoinduced Change in the Charge Order Pattern in the Quarter-Filled Organic Conductor (EDO-TTF $)_{2} \mathrm{PF}_{6}$ with a Strong Electron-Phonon Interaction," Phys. Rev. Lett. 101, 067403 (2008).

7. N. Fukazawa, M. Shimizu, T. Ishikawa, et al. "Charge and Structural Dynamics in Photoinduced Phase Transition of (EDO-TTF $)_{2} \mathrm{PF}_{6}$ Examined by Picosecond Time-Resolved Vibrational Spectroscopy," J. Phys. Chem. C, 116, 5892 (2012)

8. T. Kobayashi, D. Du, W. Feng, and K. Yoshino, "Excited-State Molecular Vibration Observed for a Probe Pulse Preceding the Pump Pulse by Real-Time Optical Spectroscopy," Phys. Rev. Lett. 101, 037402 (2008)

9. A. J. Sabbah and D. M. Riffe, "Femtosecond pump-probe reflectivity study of silicon carrier dynamics," Phys. Rev. B 66, 165217 (2002) 